\title{
A 12-Month Retrospective Comparison of the Efficacy and Complications of Device- Based and Non-Device-Based MIGS Procedures
}

\author{
Rabiah Amjad ${ }^{1}$, Louis Cantor ${ }^{1}$ \\ ${ }^{1}$ Indiana University School of Medicine, Department of Ophthalmology
}

Background: Minimally Invasive Glaucoma Surgery (MIGS) encompasses a group of procedures designed to reduce trauma to the target tissue and reduce the incidence of complications. MIGS procedures can be divided into two overall categories: device-based procedures and non-device-based procedures. The purpose of this study is to compare the efficacy and complications of device-based and non-device-based MIGS procedures in eyes combined with phacoemulsification.

Methods: 55 eyes from 36 patients with combined MIGS (iStent, GATT, ABIC, BANG, and $\mathrm{KDB}$ ) and phacoemulsification were included. The glaucoma diagnoses included primary open angle glaucoma (POAG), secondary open angle glaucoma (SOAG), and combined-mechanism glaucoma (CMG). The efficacy of the device-based and non-device-based procedures was determined by the mean postoperative IOP and glaucoma medication reduction. Early complications included adverse events and IOP spikes. Late complications included additional surgeries.

Results: At 12 months post-operatively, non-device-based eyes had a greater statistically significant reduction in IOP $(3.6 \pm 1.3 \mathrm{mmHg})$ when compared to device-based eyes $(.07 \pm 1.3$ $\mathrm{mmHg}$ ). There was not a statistically significant difference regarding glaucoma medication burden change between the non-device-based eyes $(.58 \pm .20,29.7 \%$ reduction) and the device-based eyes $(.76 \pm .19,31.7 \%$ reduction) $(p=.52)$. The occurrence of hyphema was not statistically significantly greater in device-based eyes compared to non-device-based eyes with the sample sizes in this study. The difference between IOP spikes in the two groups was not statistically significant.

Conclusion: Non-device-based MIGS procedures reduced the 12-month post-operative IOP more than the device-based MIGS procedures. There was not a statistically significant difference regarding glaucoma medication burden change between the study groups. Postoperative complications occurred at a similar frequency between the two groups.

Clinical Impact and Implications: The results of this study can help surgeons choose the appropriate MIGS procedure for their patients depending on the efficacy and safety profile. 